\title{
Streptococcus Constellatus Empyema Presenting With Undulant Fever Pattern- A Case Report and Literature Review
}

\author{
Bistees George, BS, BA ${ }^{1^{*}}$, Nouman Tanveer, $D O^{2}$ and Michael Boyars, $M D^{2}$ \\ ${ }^{1}$ School of Medicine, University of Texas Medical Branch, USA \\ ${ }^{2}$ Department of Internal Medicine, University of Texas Medical Branch, USA
}

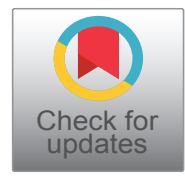

*Corresponding author: Bistees George, BS, BA, School of Medicine, University of Texas Medical Branch, 301 University Blvd, Galveston, TX 77555-0419, USA, Tel: 469-450-4169

\author{
Keywords \\ Streptococcus constellatus, Empyema, Undulant fever \\ pattern, Video-assisted thoracic surgery, Pleurodesis
}

\section{Background}

Streptococcus anginosus group (SAG), or milleri group streptococci (MGS), is an uncommon group of bacteria that has been increasingly affecting the adult population. It is an interesting organism in that it is intensely pyogenic when pathogenic-much in the same character as Staphylococcus aureus. SAG includes three species: Streptococcus anginosus, Streptococcus intermedius, and Streptococcus constellatus. Members of the SAG are usually part of the indigenous oral, gastrointestinal, and genitourinary flora; however, with mucosal disturbance they can become pathogenic leading to infections $[1,2]$. S. anginosus is commonly isolated in blood cultures, while $S$. intermedius, and S. constellatus often cause abscess formation $[3,4]$. Approximately, 1/5 of SAG infections [5] involve the chest with a mortality rate of $5-30 \%$ [6]. Thoracic infections caused by SAG usually present with the classic respiratory infections' signs and symptoms such as ill-appearance, fever, signs of respiratory distress, and hypoxemia, that usually resolve within one to two weeks with proper management. We here in present a unique case of a 49-year-old male who was found to have Streptococcus constellatus empyema, with an undulant fever pattern, needing extensive decortication with pleurodesis and a prolonged hospital course.

\section{Case Report}

A 49-year-old, Caucasian male, with an implanted loop recorder, reported having sudden onset pleuritic chest pain, shortness of breath, and a fever of $101^{\circ} \mathrm{F}$. He presented to his local emergency department (ED) where CT scan showed a non occlusive filling defect suspicious for pulmonary embolism (PE). He was transferred to our institution for further care.

Upon arrival, he received therapeutic Enoxaparin (1 $\mathrm{mg} / \mathrm{kg}$ ) for possible PE and empiric antibiotic therapy (Levaquin, Vancomycin, Piperacillin/Tazobactam) for suspected infectious etiology. On admission, patient was breathing normally, and his lungs sounded clear bilaterally on auscultation. He underwent extensive disease workup including COVID-19 testing and D-dimer. Patient was negative for COVID-19; however, he had leukocytosis and an elevated D-dimer of 2,380. CT pulmonary angiography (Figure $1 \mathrm{~A}$ ) successfully ruled out PE and showed a pleural effusion.

The next day, he was in respiratory distress, and he had decreased left lung sounds on auscultation. As the patient's condition worsened, therapeutic thoracentesis was performed, draining $750 \mathrm{ml}$ of serous fluid, and a chest tube was placed. Pleural fluid microbiology grew S. constellatus, and patient was started on cefazolin. However, he started to have an undulant fever pattern with evening fevers, leukocytosis, and worsening chest pain, shortness of breath, productive cough, and toothache. CT scan showed rapid interval worsening of the pleural effusion (Figure 1B) requiring video-

Citation: George B, Tanveer N, Boyars M (2021) Streptococcus Constellatus Empyema Presenting With Undulant Fever Pattern- A Case Report and Literature Review. Int J Respir Pulm Med 8:160. doi.org/10.23937/2378-3516/1410160

Accepted: September 27, 2021; Published: September 29, 2021

Copyright: (c) 2021 George B, et al. This is an open-access article distributed under the terms of the Creative Commons Attribution License, which permits unrestricted use, distribution, and reproduction in any medium, provided the original author and source are credited 

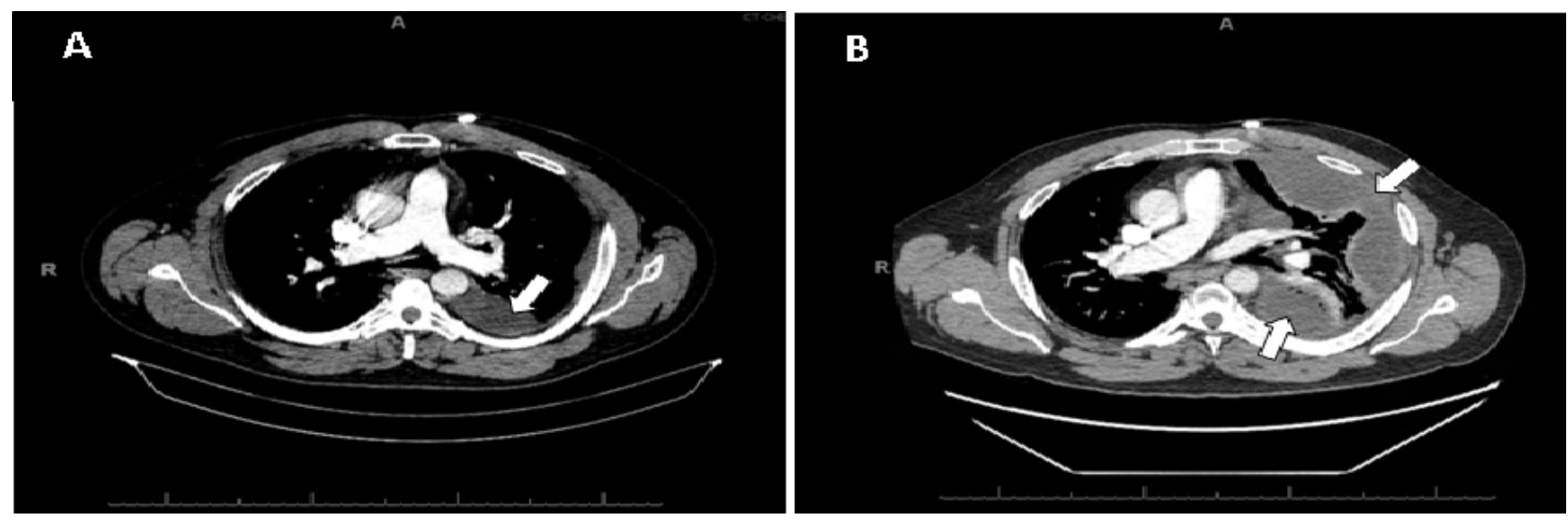

Figure 1: A) CT Scan showing moderate size multiloculated left pleural effusion; B) CT Scan showing rapid interval worsening of the left upper and lower lobe pleural effusion.
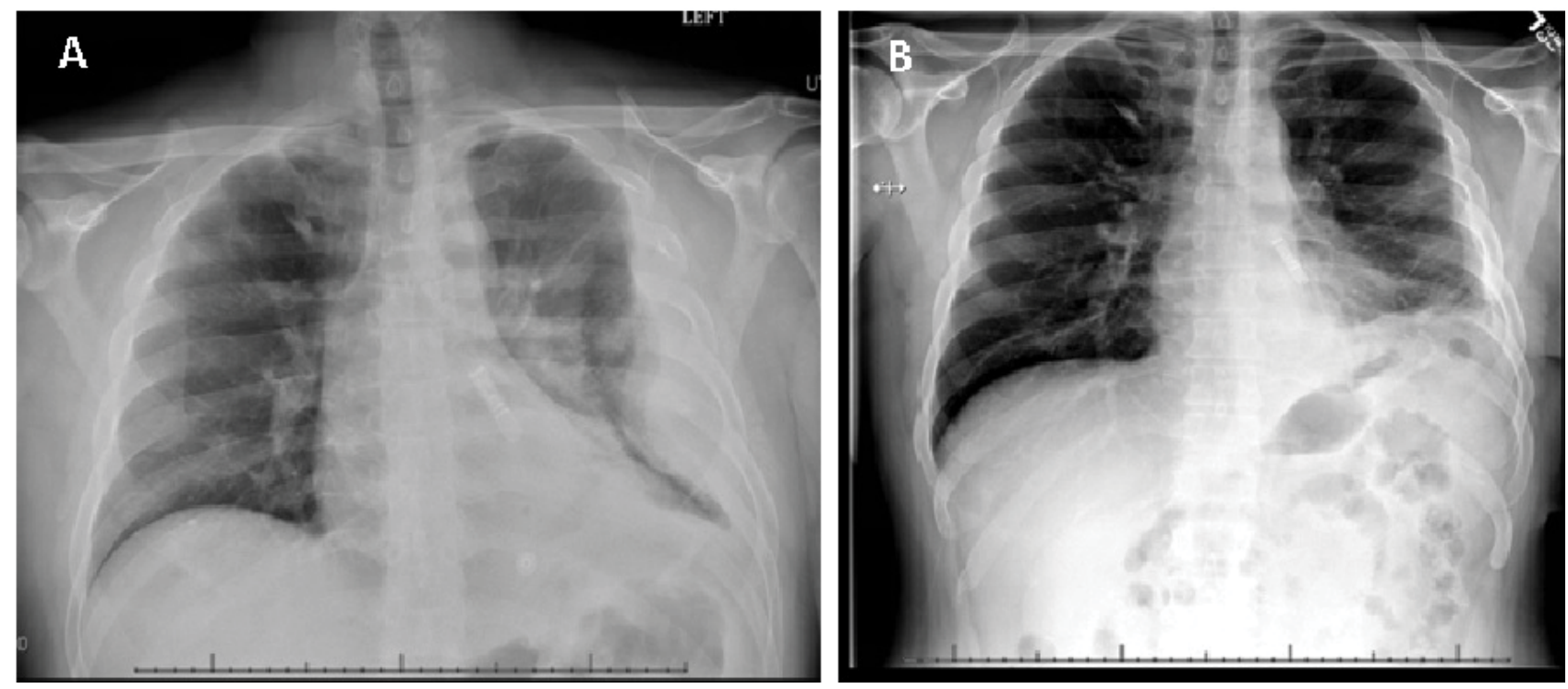

Figure 2: A) Chest X-ray obtained on admission showing a large loculated pleural effusion on the left; B) Chest X-ray performed during patient's follow up visit (one week after discharge) showing clear and expanded lungs with mild basilar subsegmental atelectasis on the left.

assisted thoracoscopic surgery (VATS) decortication with pleurodesis. Given the patient's persistent evening fevers, leukocytosis, chest pain, headaches, and toothaches, metastatic infection was on the differential. Brain MRI, CT abdomen, CT maxillofacial, and transthoracic echocardiography (TTE) were obtained. Brain MRI, CT abdomen, and TTE were unremarkable; however, images of his oral cavity were indicative of periodontal disease. As the evening fevers persisted, follow-up thoracic imaging was performed showing new development of multiloculated left empyema in left upper lobe. Chest tube was inserted to drain the new lesion, and antibiotics were broadened to Piperacillin/ Tazobactam. After a month of hospitalization, he improved and was discharged home with amoxicillin and follow up appointments with pulmonology and his dentist.

During his follow up visit, his chest X-ray (CXR) was unremarkable compared to the CXR obtained on admission (Figure 2A and Figure 2B). He also reported improving significantly with complete symptom relieve.

\section{Conclusion}

S. constellatus is becoming increasingly recognized as a cause of pulmonary infections; however, it remains to be under-reported in the literature, especially its infectious presentation. An interesting and unique aspect of our case was the patient's undulant fever pattern and prolonged hospitalization course. While caring for this patient, our team was baffled by his unique fever evening pattern presentation. The pattern was not associated with antipyretic administration. Additionally, literature review did not reveal any previous cases of SAG empyema with an undulant fever pattern (rising and falling pattern)-a term that is typically associated with Brucellosis, a zoonotic infectious disease caused by Brucella. As the patient's infection improved, his fever ceased. 
As mentioned earlier, S. constellatus is commensal in the oral cavity, gastrointestinal, and genitourinary mucosa. It usually is associated with purulent collections in the oral, thoracic, and abdominal cavities, but can also lead to serious infections such as endocarditis, intracerebral abscesses, and osteomyelitis. SAG has two different pathogenetic mechanisms. First mechanism is by the spread from a cranial site of infection such as mastoiditis, odontogenic infection, or sinusitis $[2,7]$. Second mechanism is through hematogenous spread from the respiratory or gastrointestinal tracts $[7,8]$. In our case, we believe that our patient developed the empyema secondary to his periodontal disease. It is imperative to determine the original site of infection for proper source management and determine need for further workup.

As SAG has the propensity to disseminate with mucosal disturbance, it is necessary to perform multiple organ imaging to look for foci of suppurative infections throughout the body. In our case, our patient had an implanted loop recorder, and his conditioned continued to worsen throughout his hospitalization course. We were concerned about possible hematogenous spread or distant abscess formation. Therefore, complete soft tissue imaging was performed to assess for possible disseminated infection.

Although, the incidence of SAG-related empyema is low, it remains to have high rate of morbidity and mortality rates. Therefore, it is important for clinicians to recognize how it can present to be able to provide timely diagnosis and management with antibiotics, drainage, or surgery to avoid dire outcomes [9].

\section{Competing Interests}

The authors declare that there are no conflicts of interest or any disclosure of grants or other funding regarding the submitted article.

\section{Contributors}

BG, NT, MB wrote the manuscript and reviewed the literature.

\section{References}

1. Piscitelli SC, Shwed J, Schreckenberger P, Danziger LH (1992) Streptococcus milleri group: Renewed interest in an elusive pathogen. Eur J Clin Microbiol Infect Dis 11: 491498.

2. Han JK, Kerschner JE (2001) Streptococcus milleri: An organism for head and neck infections and abscess. Arch Otolaryngol Head Neck Surg 127: 650-654.

3. Reis-Melo A, Soares D, Magalhães MF, Ferraz C, Vaz L (2020) Complicated pneumonia with empyema caused by streptococcus anginosus in a child. Rev Paul Pediatr 38.

4. Asam D, Spellerberg B (2014) Molecular pathogenicity of streptococcus anginosus. Mol Oral Microbiol 29: 145-155.

5. Madrid-Carbajal CJ, Molinos L, García-Clemente M, Pando-Sandoval A, Fleites A, et al. (2014) Pleural effusion due to streptococcus milleri: Case descriptions. Arch Bronconeumol 50: 404-406.

6. Sahn SA (2007) Diagnosis and management of parapneumonic effusions and empyema. Clin Infect Dis 45: 1480-1486.

7. Giuliano S, Rubini G, Conte A, Goldoni P, Falcone M, et al. (2012) Streptococcus anginosus group disseminated infection: Case report and review of literature. Infez Med 20: $145-154$

8. Velghe A, Van den Noortgate $N$, Janssens $W$, Smeets $P$, Vogelaers D (2004) Streptococcus milleri-sepsis with lung and brain abscesses. Acta Clin Belg 59: 369-372.

9. Azharuddin M, Prudence D, Shukla PS, Mathur A (2017) Rapid developing empyema by group $F$ beta Streptococcus anginosus group. BMJ Case Rep 2017: bcr2017219617. 